\title{
Why Ben-Naim's Deepest Pitfall Does Not Exist
}

\author{
Yi Fang \\ Department of Mathematics, Nanchang University, Nanchang, China \\ Email: yifang@ncu.edu.cn, yi.fang3@gmail.com
}

Received 2 December 2014; accepted 15 April 2015; published 17 April 2015

Copyright (C) 2015 by author and Scientific Research Publishing Inc.

This work is licensed under the Creative Commons Attribution International License (CC BY). http://creativecommons.org/licenses/by/4.0/

c) (i) Open Access

\begin{abstract}
Ben-Naim warns that there are pitfalls in pursuing Anfinsen's thermodynamic hypothesis. We show that the deepest one of his pitfalls is not a pitfall at all and the believing pursuing the minimum of the Gibbs free energy will lead us to the native structure. The "pitfall" came from the flawed inference of Gibbs energy function and the muddiness on concepts such as thermodynamic system and the second law of thermodynamics.
\end{abstract}

\section{Keywords}

Protein Folding, Gibbs Free Energy, Quantum Mechanics, Statistical Mechanics

\section{Introduction}

The abstract of [1] states that "A few pitfalls associated with the Anfinsens thermodynamic hypothesis are discussed. The most profound one is the misinterpretation of the Anfinsen's thermodynamic hypothesis? in terms of the Second Law of Thermodynamics. This misinterpretation has inspired many scientists to search for a global minimum in the Gibbs energy as a function of the conformation of the protein, sometimes referred to as the Gibbs energy landscape. Such a minimum in the Gibbs energy is different from the minimum required by the Second Law of Thermodynamics."

We will discuss "the most profound one” of Ben-Naim's pitfalls and at the end show that it is Ben-Naim’s misinterpretation of the Anfinse's threnodynamic hypothesis that produced the "pitfall".

First let us recall what is Anfinsen's thernodynamic hypothesis (it should be called thermodynamic principle, since it is nothing but the second law of thermodynamics once the spontaneousnesss of protein folding is proved). Anfinsen stated in [2] clearly that "The studies on the renaturation of the fully denatured ribonuclease required many supporting investigations to establish, finally, the generality which we have occasionally called the 'thermodynamic hypothesis'. This hypothesis states that the three-dimensional structure of a native protein 
in its normal physiological milieu (solvent, $\mathrm{pH}$, ionic strength, presence of other components such as metal ions or prosthetic groups, temperature, and other) is the one in which the Gibbs free energy of the whole system is lowest; that is that the native conformation is determined by the totality of the inter atomic interactions, and hence by the amino acid sequence, in a given environment."

Ben-Naim first pointed out in [3] that "However, since proteins live in solution, and since the solvent effects are dominant in both the dynamics of the protein folding, as well as the stability of the native structure of the protein, the relevant landscape is the GEL (Gibbs energy landscape), and not the EL (energy landscape).” This is certainly correct but really unnecessary, since the second law of thermodynamics specified that the Gibbs free energy will minimized. Continuing he states:

"The following two statements are true:

(a) The native stable structure of the protein must be at a minimum of the GEL (Gibbs Energy Landscape).

(b) Upon releasing a constraint within the system, specified by the variables: $T, P \vec{N}$, the Gibbs energy of the system, will reach a single absolute minimum.”

"The first statement (a) is essentially Anfinsen’s hypothesis... The second statement (b) is equivalent to the Second Law of thermodynamics.”

Then there is a pitfall: "From the two true statements (a) and (b), people have concluded that the stable state of the protein must be in a global minimum in the GEL. Unfortunately, this conclusion is invalid. The last statement seems to be an absurd; how can a false statement be derived from two solid, true statements? The reason so many people fell into this pitfall is that in making statements (a) and (b), we have not specified the variables with respect to which the Gibbs energy has a minimum.” [3].

Ben-Naim here clearly tells us that the pitfall comes from correctly specifying the variable of a Gibbs free energy function.

Bennaim's “the deepest pitfall” [1] is as follows:

“(iii) The third pitfall is the deepest, hence, potentially most harmful, or more dangerous to fall into. As stated in Anfinsens hypothesis: the Gibbs free energy of the whole system is lowest. That is certainly correct, but that is also trivially correct for any process or any chemical reaction. It follows from the Second Law of Thermodynamics that when a spontaneous process occurs in a system under constant temperature, pressure and composition, the Gibbs energy will be lowered, and at the new equilibrium state it will be at a minimum. This principle follows from the Second Law, and is equivalent to the statement that in an isolated system the entropy reaches its maximum. The question is, maximum of entropy with respect to what, or minimum of Gibbs energy with respect to what? If you walk along a rough road and you are not careful, you might fall into a pitfall. Why?”

So the problem is still correctly identifying the "whole system" and formulate the Gibbs free energy function with coreect variable. What is this function? Ben-Naim shows his reason in [1] and [3], in an example, For a system characterised by the variables $T, P$, and $N$, we can write the Gibbs energy function of the system as $G(T, P, N ; \vec{R})$.

"If we start with a system having one particle at a fixed position, say $\vec{R}=\vec{R}_{0}$, then releasing the constraint on $\vec{R}$, but keeping $T, P$ and $N$ fixed, the system's Gibbs energy will always decrease by the amount:

$$
\Delta G=k_{B} T \ln \rho \Lambda^{3}<0 ” .
$$

This argument has a fundamental flaw, because with a little mathematics, it infers that:

$$
\liminf _{\vec{R} \rightarrow \vec{R}_{0}} G(T, P, N ; \vec{R})=-\infty .
$$

This is indeed a very bad function, not only it is discontinuous everywhere, but also it cannot have any finite minimum value. No such function can be an energy function in physics. No matter what is the reason, this inference just points out that something was wrong in Ben-Naim's argument. Ben-Naim pointed out that the $\vec{R}$ is only a point in $\mathbb{R}^{3}$, not a conformation in [4]. But in [3] he also states that:

"The case of protein folding has the same pitfall as in the relatively simple examples discussed above. The GEL function is $G\left(T, P, N ; \Phi_{1}, \Psi_{1}, \cdots, \Phi_{M}, \Psi_{M}\right)$ where the configuration of the entire protein is given by the 2M dihedral angles $\Phi_{1}, \Psi_{1}, \cdots, \Phi_{M}, \Psi_{M}$. Starting from any initial configuration $\Phi_{1}^{\text {in }}, \cdots, \Psi_{M}^{\text {in }}$, (say, of the unfolded form), the Gibbs energy function has the value $G\left(T, P, N ; \Phi_{1}^{\mathrm{in}}, \cdots, \Psi_{M}^{\mathrm{in}}\right)$. When we release the constraint of a fixed configuration, the Gibbs energy of the system will reach a new minimum; it is not a minimum 
with respect to the variables $\Phi_{1}, \Psi_{1}, \cdots, \Phi_{M}, \Psi_{M}$ but with respect to all possible distributions $P\left(\Phi_{1}, \Psi_{1}, \cdots, \Phi_{M}, \Psi_{M}\right)$ i.e. the Gibbs energy functional has a minimum at some distribution $P_{\text {eq }}\left(\Phi_{1}, \Psi_{1}, \cdots, \Phi_{M}, \Psi_{M}\right)$ with value of $G\left(T ; P ; N ; P_{\text {eq }}\left(\Phi_{1}, \Psi_{1}, \cdots, \Phi_{M}, \Psi_{M}\right)\right)$.”

So the answer to his question of "minimum of Gibbs energy with respect to what?" is certainly not the conformation $\Phi_{1}^{\text {in }}, \cdots, \Psi_{M}^{\text {in }}$. Therefore, if one wants to solve the minimisation problem

$$
G\left(T, P, N ; \Phi_{1}^{\min }, \Psi_{1}^{\min }, \cdots, \Phi_{M}^{\min }, \Psi_{M}^{\min }\right)=\min _{\text {All of conformations }} G\left(T, P, N ; \Phi_{1}, \Psi_{1}, \cdots, \Phi_{M}, \Psi_{M}\right),
$$

Then one falls into "the deepest pitfall". The reason is not clearly given, if he thinks that the function of $G\left(T, P, N ; \Phi_{1}, \Psi_{1}, \cdots, \Phi_{M}, \Psi_{M}\right)$ will have the same property as in (2),

$$
\liminf _{\Phi_{1}, \Psi_{1}, \cdots, \Phi_{M}, \Psi_{M} \rightarrow \Psi_{1}^{\text {in }}, \cdots, \Phi_{M}^{\text {in }}, \Psi_{M}^{\text {in }}} G\left(T, P, N ; \Phi_{1}, \Psi_{1}, \cdots, \Phi_{M}, \Psi_{M}\right)=-\infty,
$$

he is definitely wrong as we have already expained. Except this, he did mention that the second law of the thermodynamics cannot guarantee that the Gibbs free energy will have a global minimum if the variable is the conformation [3]. Without a concrete formula of the Gibbs free energy function $G\left(T, P, N ; \Phi_{1}, \Psi_{1}, \cdots, \Phi_{M}, \Psi_{M}\right)$, this argument certainly has no weight at all. On the contrary, general mathematical theorems tell us in many circumstances a minimiser of a function can always be found, for example, if the function is lower semicontinues and is defined on a compact set. Nevertheless, Ben-Naim still states that pursuing solving (3) will lead to his "deepest pitfall". Hiis advice of avoiding this "pitfall" is to adapt the Gibbs free energy functional $G\left(T ; P ; N ; P\left(\Phi_{1}, \Psi_{1}, \cdots, \Phi_{M}, \Psi_{M}\right)\right)$, the variable is not the conformation $\Phi_{1}, \Psi_{1}, \cdots, \Phi_{M}, \Psi_{M}$, but the probability distributions of an ensemble of conformations, $P\left(\Phi_{1}, \Psi_{1}, \cdots, \Phi_{M}, \Psi_{M}\right)$.

Thus we may say that Ben-Naim actually falls into a pitfall: he thinks that only by considering an ensemble of conformations we can apply the second law of thermodynamics.

In fact, the Gibbs free energy function $g\left(X ; E_{N}\right)$, where $X$ is a conformation of a protein and $E_{N}$ is the physiological environment of the protein in nature, does exist, and is explicitly derived via quantum statistics by considering a thermodynamic system consisting of a single conformation and its immediate environment, see [5], [6], [7], and [8]. Minimizing $g\left(X ; E_{N}\right)$ is actually applying the second law of thermodynamics, but not to an ensemble of conformations.

So the "deepest pitfall" of Ben-Naim claimed comes from flawed inferences, misunderstanding of Anfinsen's thermodynamic hypothesis, and prejudice about how to apply the second law of thermodynamics.

\section{Two Kinds of Thermodynamic Systems and Two Gibbs Free Energies}

If Ben-Naim noticed that there are actually two kids of thermodynamic systems, single molecule and ensemble, and if he uses the single molecule one in his point (a) and (b) and apply the second law of thermodynamics, he would not have claimed "the deepest pitfall".

In fact, to generate the GEL, there should be a Gibbs free energy function $g(X ; E)$ of a thermodynamics system $\mathcal{T}_{X}$ determined by the conformation $X$. In [5], [6], [7], and [8], we carefully delimited the boundary of $\mathcal{T}_{X} \subset \mathbb{R}^{3}$ such that it is tailor made for the conformation $X$. Here $X=\left(x_{1}, \cdots, x_{N}\right) \in \mathbb{R}^{3 N}$ are the atomic centers of all atoms $\left(a_{1}, \cdots, a_{N}\right)$ of a protein $\mathfrak{U}$, and $\mathcal{T}_{X}$ in fact is $P_{X}=\bigcup_{i=1}^{N} B\left(x_{i}, r_{i}\right)$ plus the first layer of water molecules surrounding $P_{X}$; $E$ represent environment, such as Ben-Naim's $T, P, N$, and may be a little more.

Hence $g(X ; E)$ is the Gibbs free energy of the thermodynamic system $\mathcal{T}_{X}$. Note that $\mathcal{T}_{X}$ changes with $X$, we have as many thermodynamic systems as possible of conformations $X$. The second law of thermodynamics is applied to a series thermodynamic systems until at last almost achieves the minimum Gibbs free energy.

We will use $E_{N}$ to denote the physiological environment as stated in [2]. Just thinking that watching an individual protein folding from an extended conformation to near by the native structure, by Anfinsen hypothesis (or just a version of the second law), it must be 


$$
g\left(X_{N} ; E_{N}\right)=\min _{\text {All conformations } X} g\left(X ; E_{N}\right),
$$

where $X_{N}$ is the native structure. Or, in case that $X_{N}$ is only a local minimum (in general it is possible that $X_{N}$ may be just a local minimum, as pointed out by Ben-Naim in [3], what we should emphasised is that in this situation, the initial conformation $X_{0}$ becomes very important, different $X_{0}$ may lead to different minimisers.)

$$
\nabla g\left(X_{N} ; E_{N}\right)=\overrightarrow{0} .
$$

This is a single molecule version of the Gibbs free energy. If known, then for the $a b$ initio prediction of protein structure, $g\left(X ; E_{N}\right)$ gives a firm theoretical base of the emerging theory of protein folding based on the fundamental second law of the thermodynamics.

Such an analytic function $g(X ; E)$ actually exists. In [5], [6], [7] and [8] it was deverived via the quantum statistic mechanics for monomoric globular proteins:

$$
g\left(X ; E_{N}\right)=v_{e} \mu_{e} V\left(\Omega_{\mathbf{X}}\right)+d_{w} v_{e} \mu_{e} A\left(M_{\mathbf{X}}\right)+\sum_{i=1}^{H} v_{i} \mu_{i} A\left(M_{\mathbf{X} i}\right)+\sum_{A \neq B} \frac{q_{A} q_{B}}{\left|x_{A}-X_{B}\right|} .
$$

For the meaning of terms in (7) please see [8]. The derivation heavily depends on the global geometry of $\mathcal{T}_{X}$ and various hydrophobic levels of moieties of amino acids appearing in the amino acid chain of the protein. The spacial distribution of atomic centres of these hydrophobic levels change with conformation change, is a rough representation of electron density of wave functions corresponding to the conformation $X$. For globular proteins we can assume that the environment $E$ contains only water molecules. For non-globular proteins, the environment $E_{N}$ will be much more complicated, we have not been able to find a way to make similar derivations. We guess that the corresponding $g\left(X ; E_{N}\right)$ may have more complicated form than (7).

Now consider a system of $M$ conformations of the same protein, $X_{1}, \cdots, X_{M}$, this is the system Ben-Naim chooses to apply the second law of thermodynamics. This ensemble case is more familiar, and in fact is the only thermodynamic system appeared in protein folding literature so far. Single molecule phenomena, such as what is the shape of native structure, can never be deduced from such an ensemble system. Instead, the concern should be concentrated on knowing the collective phenomena such as folding rate and folding time, etc. Then indeed we should consider the distribution $P\left(X_{1}, \cdots, X_{M} ; E_{N}\right)$. For example, the second law of thermodynamics indicates that there is a distribution $P_{\text {eq }}\left(X_{1}, \cdots, X_{N} ; E_{N}\right)$ such that when the proteins at full function state, for any distribution $P$, it will be

$$
G\left(P_{\text {eq }}\left(X_{1}, \cdots, X_{M}\right) ; E_{N}\right) \leq G\left(P\left(X_{1}, \cdots, X_{M}\right) ; E_{N}\right) .
$$

The problem is, nobody knows $P_{\text {eq }}$, as Ben-Naim admitted in [4]. One suggestion is that we apply $g\left(X ; E_{N}\right)$ and try the Boltzmann distribution

$$
P_{\text {eq }}\left(X_{i} ; E_{N}\right)=\frac{\exp \left(-\frac{g\left(X_{i} ; E_{N}\right)}{k T}\right)}{\sum_{j=1}^{M} \exp \left(-\frac{g\left(X_{j} ; E_{N}\right)}{k T}\right)} .
$$

Because that any conformation $X$ such that $g\left(X ; E_{N}\right) \gg g\left(X_{N} ; E_{N}\right)$ will have much smaller $P_{\text {eq }}\left(X ; E_{N}\right)$, thus less chance to appear in the full function state of the ensemble. Of course, this is only a conjecture and it is not so important to know, at least not as Ben-Naim claimed in [4]: "If one knew this distribution, then one could tell which conformations are more probable than the others under the given environment." Okay, we now all know that in physiological situation, the native structure is "more probable than the others under the given environment", but, just by this knowledegy, could you tell me what is the geometric shape of the native structure? So to solve the protein folding problem, at least for the prediction of native structure from the knowledge of amino acid sequence, we have to know what is $g\left(X ; E_{N}\right)$ and solve (5) or (6) with whatever mathematical method. That is, we should pursue the "deepest pitfall" to solve the protein folding problem, because it is not a pitfall at all. 


\section{Conclusion}

We have demonstrated that the "deepest pitfall” of Ben-Naim claimed is not a pitfall at all. Because of lack of mathematical skill, misconnect of second law of thermodynamics can only be applied to ensemble systems, and moreover, faille of deriving a Gibbs free energy formula $g(X ; E)$ of portion folding from the underline principle of Anfinsen's thermodynamic hypothesis, Ben-Naim wrongly warned people that pursuing the thermodynamic hypothesis will lead them to "pitfall".

\section{References}

[1] Ben-Naim, A. (2011) Pitfalls in Anfinsen’s Thermodynamic Hypothesis. Chemical Physics Letters, 511, 126-128. http://dx.doi.org/10.1016/j.cplett.2011.05.049

[2] Anfinsen, C.B. (1973) Principles That Govern the Folding of Protein Chains. Science, 181, 223-230. http://dx.doi.org/10.1126/science.181.4096.223

[3] Ben-Naim, A. (2012) Levinthal's Question Revisited, and Answered. Journal of Biomolecular Structure and Dynamics, 30, 113-124. http://dx.doi.org/10.1080/07391102.2012.674286

[4] Ben-Naim, A. (2013) Comment on a Paper: “Ben-Naim’s ‘pitfalls’: Don Quixote’s windmill”, by Y. Fang, Open Journal of Biophysics, 2013, 3, 13-212. Open Journal of Biophysics, 3, 275-276. http://dx.doi.org/10.4236/ojbiphy.2013.34032

[5] Fang, Y. (2012) Gibbs Free Energy Formula for Protein Folding. In: Morales-Rodriguez, R., Ed., Thermodynamics Fundamentals and Its Application in Science, InTech, 47-82.

http://www.intechopen.com/books/thermodynamics-fundamentals-and-its-application-in-science http://dx.doi.org/10.5772/2615

[6] Fang, Y. (2013) Ben-Naim’s “Pitfalls”: Don Quixote’s Windnmill. Open Journal of Biophysics, 3, 13-21. http://dx.doi.org/10.4236/ojbiphy.2013.31002

[7] Fang, Y. (2014) The Second Law, Gibbs Free Energy, Geometry, and Protein Folding. Journal of Advances in Physics, 3, 278-285.

[8] Fang, Y. (2014) A Gibbs Free Energy Formula for Protein Folding Derived from Quantum Statistics. Science China, Pysics, Mechanics \& Astronomy, 57, 1547-1551. http://dx.doi.org/10.1007/s11433-013-5288-x 